\title{
EL CONCEPTO DE COMPETENCIA DE ALF ROSS *
}

\author{
Robert Alexy \\ Universidad de Kiel
}

RESUMEN. Con base en el trabajo pionero de A. Ross, el concepto de competencia es definido en este artículo a través de cuatro características. Dichas características son normatividad, potencialidad, declaratividad y dispositividad. Según esta definición, una competencia es la posibilidad normativa de cambiar una posición jurídica a través de una declaración dirigida a este objetivo. De manera ulterior se analiza además la tesis rossiana de la reducción, según la cual todo concepto normativo puede ser reducido al operador "O». En este artículo se intentará mostrar que la tesis de Ross es correcta, dado que las competencias pueden ser entendidas como un deber ser potencial.

Palabras clave: A. Ross, competencia, deber ser como modalidad deóntica fundamental, problema de la reducción, competencia como deber ser potencial.

ABSTRACT. Based on A. Ross' groundbreaking work, the concept of power is defined in this article through four characteristics. These are normativity, potentiality, declarativity and disposability. According to this definition, power is the possibility of changing a legal position by means of a statement, which is aimed to this goal. Subsequently is analysed the reduction thesis, which was proposed by Ross. According to this thesis, any normative concept can be reduced to the operator "O". This article tries to show that Ross' thesis is correct, due to the fact that power can be understood as a potential ought.

Keywords: A. Ross, competence, power, ought as basic deontic modality, reduction problem, power as potential ought.

* Fecha de recepción: ¿? de ¿? de 2012. Fecha de aceptación: ¿? de ¿? de 2012.

Traducción a cargo de G. VILLA Rosas del original en alemán «Alf Ross' Begriff der Kompetenz», publicado en Gedächtnisschrift für Jörn Eckert, 15.Mai 1954 bis 21.März 2006, 2008, 43-64. El traductor agradece a A. SANTACOLOMA SANTACOLOMA la corrección de la versión preliminar en español de este escrito. 


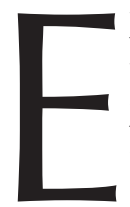

1 concepto de competencia es no sólo una controvertida categoría sistemática de la teoría general y de la filosofía del derecho, sino una herramienta jurídica comúnmente utilizada. No obstante, la terminología que se refiere a ella es variada. Otras expresiones usadas para referirse a ella son «habilitación» ${ }^{1}$ (Ermächtigung) y, en inglés «power». A pesar de semejantes diferencias terminológicas existe un acuerdo extendido, más o menos uniforme, acerca de los fenómenos que pueden ser descritos como ejercicios de una habilitación o, como se debería decir mejor, de una competencia. En el derecho privado, la celebración de contratos, el matrimonio y el otorgamiento de testamento son casos paradigmáticos de competencia. La promulgación de leyes y sentencias, así como el decreto de actos administrativos son los ejemplos más citados en el derecho público. Los juristas están familiarizados con todo esto y, por lo general, los problemas que tienen con los ejercicios de competencia presuponen el hecho mismo de que se trata de ejercicios de competencia. Ellos no parecen, sin embargo, sentirse familiarizados con la pregunta misma acerca de lo que son competencias. La respuesta a esta pregunta se vuelve aún más difícil, si se comienza a leer lo que han escrito los teóricos del derecho sobre este tema. Ésta es una señal clara acerca de la existencia de un problema de índole filosófica. Un concepto genera en la vida cotidiana pocos o ningún problema; pero si éste se comienza a analizar, resulta del todo dudoso. La razón fundamental por la cual esto sucede, radica en el hecho de que el concepto de competencia está relacionado de manera intrínseca con otros conceptos jurídicos fundamentales, como son aquellos de norma, validez, obligación, derecho subjetivo, autoridad y autonomía. El análisis del concepto de competencia debe ser por esto un análisis de una red de conceptos, y dado que todos estos conceptos son fundamentales, una teoría de la competencia debe incluir los elementos esenciales de una teoría sobre la naturaleza del derecho.

${ }^{1}$ N. del T.: Para efectos de esta traducción al castellano se ha preferido la palabra «habilitación» sobre otros usos en castellano correspondientes a la palabra germánica «Ermächtigung», por las siguientes razones. Primera, en relación al término germánico «Ermächtigung» en traducciones al castellano anteriores especialmente de los textos de KELSEN, es corriente el uso de otras expresiones tales como «facultamiento» [vid., por ejemplo, R. WALTER, «Origen y desarrollo del concepto de norma fundamental», Revista de la Facultad de Derecho de México, 244 (2005), 285-301, 298]. Esta expresión en particular parece demasiado forzada en el castellano usual. De manera específica, este término no se encuentra registrado en el Diccionario de la Real Academia de la Lengua Española [Diccionario de la Real Academia de la Lengua Española, 22." ed. (Madrid: Real Academia de la Lengua Española, 2001), versión online: www.rae.es]. Segunda, otros términos como facultad jurídica [vid., por ejemplo, S. L. PAULSON, «La reconstrucción radical kelseniana de la norma jurídica», en La Teoría del Derecho de Hans Kelsen, L. Clérico y J. SiECKMANn (eds.) (Bogotá: Universidad Externado de Colombia, 2011) 11-44] — «legal ability»; «Rechtsfähigkeit»— son demasiado generales, pues no aluden al fenómeno especifico del que se trata [sobre la diferencia entre «Fähigkeit» y «Ermächtigung», vid., por ejemplo, H. KeLSEN, «Recht und Kompetenz: Kritische Bemerkungen zur Völkerrechtstheorie Georges Scelles», en KeLSEn, Auseinandersetzungen zur Reinen Rechtslebre, K. RINGHOFER y R. WALTER (eds.) (Viena: Springer, 1987), 72]. Tercera, se ha preferido la voz «habilitación» sobre la palabra «autorización», debido al hecho de que el término germánico «Ermächtigung» es más cercano en castellano a la sustantivación del verbo «habilitar» que aquélla del verbo «autorizar», en la medida en que el primero ha sido definido como «hacer a alguien o algo hábil, apto o capaz para una cosa determinada» y el segundo como «dar o reconocer a alguien la facultad o el derecho para hacer algo» [Diccionario de la Real Academia de la Lengua Española, 22.. ed. (Madrid: Real Academia de la Lengua Española, 2001) versión online: www.rae.es]. Cuarta, existen usos homólogos de la voz castellana «habilitación» en el contexto jurídico de otras lenguas romances tales como el francés [vid. G. ToussEAu, Normes d'babilitation (Paris: Dalloz, 2006)]. Advirtiendo la pluralidad de términos que se refieren al fenómeno en cuestión, el autor ha preferido la utilización con pretensiones unívocas de la voz alemana «Kompetenz» que será traducida para efectos de este texto como «competencia». 
Esto aclara la razón por la cual, el concepto de competencia ha cautivado a tantos eminentes teóricos del derecho. Uno de ellos es el danés A. Ross, quien es uno de los grandes filósofos jurídicos del siglo XX. Su contribución es uno de los más interesantes intentos por comprender este huidizo concepto. En este punto uno debe preguntarse, hasta dónde ha llegado Ross y si es posible ir aún más lejos.

Los múltiples problemas vinculados al concepto de competencia pueden ser divididos en dos grupos. Los problemas del primer grupo se refieren a la pregunta acerca de qué son competencias y cómo pueden ser éstas diferenciadas de otros fenómenos similares. Esto se puede denominar, en una antigua e indeseada terminología, como el problema de la naturaleza de la competencia («Problem der Natur»). El segundo grupo de problemas apunta a la pregunta acerca de si el concepto de competencia puede ser reducido a otros conceptos, y especialmente, al concepto de obligación. Este problema debe ser denominado como el «problema de la reducción» («Reduktionsproblem»). La parte más interesante de la teoría de la competencia de Ross concierne al problema de la reducción. Sin embargo, comenzaré con algunas observaciones acerca del problema de la naturaleza. Si no se tiene claridad acerca de aquello a lo que Ross se refiere cuando habla de competencia, no se puede tener una discusión fructífera acerca de su teoría de la reducción. Me concentraré en la tardía versión de Ross de su teoría de la competencia publicada en su obra Directives and Norms de 1968. La versión anterior, que expuso Ross en su libro On Law and Justice de 1958, será tenida en cuenta no sólo con el fin de mejorar la interpretación de la más moderna, sino también y principalmente, para probar la tesis de E. BuLYGin, según la cual Ross cambió radicalmente de opinión acerca de la reducción entre 1958 y $1968^{2}$.

\section{LA NATURALEZA DE LA COMPETENCIA}

\subsection{Posibilidad}

Ross nos ofrece una definición explícita de competencia tanto en 1968 como en 1958. Según esta última:

Competence is the legally established ability to create legal norms (or legal effects) through and in accordance with enunciations to this effect ${ }^{3}$.

Según esta definición, el género próximo es el concepto de capacidad («ability»). Capacidad es un caso especial de posibilidad. Se puede decir entonces que la definición de competencia de Ross se basa en uno de los conceptos más generales y fundamentales: en la categoría de posibilidad. Esta definición debe ser denominada como la «tesis de la posibilidad» («Möglichkeitsthese»). Según esta tesis, tener una competencia significa tener una cierta clase de posibilidad.

\footnotetext{
2 Bulygin, On Norms of Competence, Law and Philosophy, 11 (1992), 201, 208.

3 Ross, Directives and Norms, 1968, 130. En adelante será referido este libro en el texto a través del año de impresión y del número de página.
} 


\subsection{Normatividad}

Existen diferentes formas de posibilidad. Ross describe las características especiales de la competencia como posibilidad a través de tres diferencias específicas. La primera consiste en la particularidad de estar jurídicamente fundada («legally established», 1968, 130). Competencias en tanto posibilidades son por ello posibilidades normativas. La primera diferencia específica es por tanto la normatividad.

Según Ross, las competencias se fundan jurídicamente en normas de competencia. Esto conduce a tres problemas. El primero resulta de la definición rossiana de norma. Según Ross, el concepto de norma incluye el concepto de validez (1968, 79 ff., 93). Si el concepto de competencia, en su definición, está vinculado al concepto de norma, entonces el concepto de validez está también incluido en el concepto de competencia. Esto significaría que nosotros podríamos hablar sólo de competencias que se apoyan sobre normas válidas, es decir, sólo de competencias existentes. Una proposición como «la competencia x no existe» incluiría una contradicción, pues según las definiciones de Ross, la competencia x no podría al mismo tiempo ser y no existir. De la misma forma no tendría sentido una proposición como «nosotros deberíamos introducir la competencia x», pues según las definiciones de Ross, si x es una competencia, entonces ella se encuentra necesariamente ya en vigor. Pero, ni esta proposición carece de sentido, ni la primera es contradictoria. Es por ello inadecuado incluir en el concepto de competencia aquél de validez. Una competencia debería ser considerada como una entidad semántica. Esto corresponde al concepto semántico de norma ${ }^{4}$, según el cual normas son contenidos semánticos (Bedeutungsgehalte), es decir directivas en el sentido de Ross $(1968,36)$. A pesar del hecho de que esta concepción semántica del concepto de norma es expresamente rechazada por Ross $(1968,80)$, una concepción semántica de la competencia se ajusta mejor que cualquier otra definición que incluya elementos de validez a su caracterización de competencias como «modalities» (1968, 118-119) o como «linguistic vehicles through which the directive content of legal rules is expressed» $(1968,134)$.

Esto conduce al segundo problema de la primera diferencia específica. Si debiese permanecer la característica juridicidad («legally») en la definición, sólo las competencias jurídicas serían posibles. Sin embargo, esto sería incompatible con la propia teoría de Ross. En el $\$ 29$ de Directives and Norms, Ross muestra que la modalidad de la competencia puede ser también aplicada a discursos normativos no jurídicos. Como ejemplo, Ross aduce el acto institucional de la promesa $(1968,135$ 136). Es recomendable por esto, proponer tres cambios en la definición. El primero, sustituir «legally established» por «normatively established»; el segundo, cambiar «legal norms» por «norms»; y el tercero, sustituir «legal effects» por «normative effects». Competencias jurídicas, morales y convencionales se presentan entonces como casos especiales de competencias normativas. Lo anterior encaja bien con la caracterización de las competencias como modalidades normativas («normative») $(1968,118,135)$.

\footnotetext{
${ }^{4}$ Comp. Alexy, Theorie der Grundrechte, 3. ${ }^{a}$ ed., 1996, $42-47$.
} 
Con respecto a la definición de Ross, uno se puede preguntar acerca de si es afortunada la calificación de la competencia como una capacidad normativamente fundada («established»). ¿No está esta característica ya contenida en la expresión «to create norms»? La respuesta a esta pregunta conduce al tercer problema de la primera diferencia específica, el cual tiene una importancia fundamental para la naturaleza de la competencia jurídica. Hay dos maneras de concebir las competencias jurídicas: una interna y otra externa. Según el concepto interno de la competencia jurídica, la existencia de una competencia presupone siempre una norma, en la cual esta competencia se basa. Esto y no otra cosa es lo expresado a través de la modificación de la característica «normativamente fundada» («normatively established»). Lo anterior puede denominarse como la «tesis normativa» («Normativitätsthese»). Competencias como posibilidades normativas son posibilidades que se fundan en normas, las cuales pueden ser jurídicas o no. En este sentido, en los términos de la normatividad, las competencias tienen un carácter sistemático interno. Esta normatividad no surge del objeto de la competencia, el cual es descrito por Ross a través de las palabras «to create legal norms (or legal effects)». Esta cláusula podría ser también relevante en el caso en el cual sólo exista capacidad o posibilidad meramente fáctica de crear normas jurídicas. En este caso no se apoyaría la competencia sobre una norma que pertenezca al sistema. La competencia sería en este sentido externa.

Uno se puede preguntar, si las competencias externas son competencias jurídicas ${ }^{5}$. Yo opino que Ross está en lo cierto cuando afirma que las competencias se apoyan siempre en normas, que son constitutivas de ellas $(1968,130)$; lo que significa que las competencias meramente externas, ya sea que existan o no, jamás son competencias jurídicas. Esta pregunta, por cierto, puede ser aquí tan sólo identificada pero no discutida. Ella conduce al problema del fundamento último del ordenamiento jurídico ${ }^{6}$, es decir, al problema de la norma fundamental. Aquí basta subrayar que la característica «normativa», como característica específica de la competencia, no está como tal contenida en la característica definitoria «to create legal norms (or legal effects)» y que ella es necesaria, si se quiere tener un concepto normativo de competencia que esté claramente separado del concepto de poder fáctico.

\subsection{Dispositividad}

La segunda diferencia específica ya ha sido mencionada en varias ocasiones. Ross en su definición se ha referido a ella al hablar de la capacidad de crear normas (o efectos jurídicos) [«to create legal norms (or legal effects)», 1968, 130]. De acuerdo con lo dicho anteriormente, esta cláusula debería ser modificada como «to create norms (or normative effects)» con el fin de lograr una mayor generalización. Pero claro, esto es de poca importancia. Una pregunta mucho más interesante es, si esta cláusula es la mejor descripción del objeto de la competencia.

5 Comp. RAz, Voluntary Obligations and Normative Powers, The Aristotelian Society, Supplementary vol. 46 (1972), 79, 83 ss. RAZ defiende la tesis según la cual «that legal powers may exist even if not conferred by any norm, whether legal or non-legal».

${ }^{6}$ Comp. SpaAK, The Concept of Legal Competence, 1994, 170; RuIter, Institutionelle Rechtstatsachen, 1995,94 y ss. 
Si se quiere definir el objeto de la competencia de la manera más abstracta posible, debería decirse que consiste en el cambio ${ }^{7}$ de una situación normativa ${ }^{8}$. El cambio de una situación normativa puede ser descrito de dos formas. Según la primera, el cambio de una situación normativa es el cambio de normas individuales o generales; según la segunda, es un cambio de posiciones normativas ${ }^{9}$. T. SPAAK ha afirmado que esta segunda forma es mejor, porque es posible cambiar posiciones jurídicas de una forma diversa a aquella que ocurre por el cambio de normas ${ }^{10}$. Este autor cita varios ejemplos. El más interesante es el de la oferta. Una oferta como tal no fundamenta obligación alguna, y en esta medida, ninguna norma obligatoria individual. No obstante, la posición del destinatario de la oferta cambia. Él se encuentra ahora en la posición en virtud de la cual, puede crear una obligación en cabeza del oferente mediante la aceptación de la oferta. Esto no es sin embargo ningún argumento que permita sostener que el concepto de posición sea más amplio que aquél de norma. Si se acepta que no sólo existen normas obligatorias sino normas de competencia, entonces se tiene únicamente que aplicar la diferencia entre normas individuales y generales no sólo a las normas obligatorias, sino también a las normas de competencia, para obtener una congruencia extensional completa del concepto de norma y del concepto de posición. Esto justifica la tesis según la cual no existe posición sin normas que a ella correspondan y no existe norma sin posiciones que a ella sean correspondientes ${ }^{11}$. Las normas del derecho de contratos fundamentan la autonomía privada como una competencia general. Una oferta concreta fundamenta la competencia individual o especial; mediante la aceptación de la oferta surge una obligación contractual individual o especial. No hay diferencia sustancial alguna si se describe esto como una posición individual o especial, o como una norma de competencia individual o especial.

Si describir el objeto de la competencia como norma o como posición no hace diferencia alguna, entonces la segunda diferencia específica de Ross no puede ser falsa. Se puede en el mejor de los casos decir que la cláusula «(or legal effects)» es redundante. Parece que Ross ha acogido esta cláusula para garantizar que pudiera ser incluido en su definición todo cambio de una situación o posición jurídica. Por ejemplo, la derogación de una ley puede ser difícilmente descrita como la creación o establecimiento de una norma. Por el contrario, dicha derogación es comprensible con ayuda del concepto de la producción de un efecto jurídico. La desventaja de la cláusula del efecto jurídico es que cada cambio de posición jurídica es un efecto jurídico. La cláusula del efecto jurídico no está por consiguiente, al mismo nivel que la cláusula del establecimiento de normas. Dicha cláusula incluye a esta última porque tiene un mayor grado de abstracción. Si la intención de Ross fue la de aclarar que cosas como la posición del destinatario de una oferta están incluidas en su definición, entonces esto puede ser expresado mejor, si la noción de efecto se reemplaza por el concepto de posición. Si se

7 El concepto de cambio de una situación normativa $S$ por una persona a a través del acto $A$, que representa un ejercicio de competencia, debe ser definido como sigue: a cambia $S$ a través de $A$ si y sólo si $S$ sería otra, si $A$ no hubiese sido realizado. Esta definición permite también evitar que una variación de la situación normativa, que se produzca sin la mediación de un ejercicio de competencia, sea interpretada como un cambio.

8 Comp. Alexy (p. 4), 212.

9 Comp. Lindahl, Position and Change, 1977, 85 y ss.

10 SPAAK (p. 6), 21.

11 Alexy (p. 4), 163 y ss. 
reúne lo que se ha dicho en relación con la segunda diferencia específica, se obtiene la siguiente formulación: «to change norms and positions». Tener la posibilidad de cambiar normas y posiciones, significa poder disponer sobre ellas. Si se quiere describir de manera sucinta la segunda diferencia específica, se puede por tanto hablar de «dispositividad» («Disposivität»).

\subsection{Declaratividad}

La reconstrucción de la definición de Ross ha conducido a definir competencia como la posibilidad normativa de disposición sobre normas y posiciones. Esto es suficiente para diferenciar la competencia de los meros permisos $(1968,131)^{12}$, pero no para solucionar el decisivo problema de la delimitación. Este problema resulta de la posibilidad de cambiar la posición jurídica a través de actos que no pueden ser adecuadamente descritos como ejercicios de competencia. El principal ejemplo es aquel que fue denominado por H. KELSEN como «capacidad delictual» («Deliktsfähigkeit») ${ }^{13}$. Esta expresión designa la capacidad de cambiar una posición jurídica a través de un acto no permitido o de un delito. Si $a$ comete un acto no permitido con respecto a $b$, cambia ambas posiciones jurídicas. $a$ responde desde este momento por el daño, y $b$ tiene una pretensión de indemnización del daño correspondiente. En el derecho penal con el acaecimiento del delito ocurre un cambio fundamental en la relación jurídica entre el autor del delito y el fiscal. En la literatura reciente han sido también mencionados ejemplos de otras áreas diversas a aquéllas del derecho de daños y del derecho penal. J. RAz debate el caso de un cambio de «application of various tax laws and one's entitlement to various social services» a través del cambio de «one's place of permanent residence from one town to another» ${ }^{14}$. N. MACCORMICK presenta el caso de una persona que comienza a producir bienes de consumo y con ello es sometida a un especial «duty of care» ${ }^{15}$. Todos estos casos tienen en común con el ejercicio de competencia, que la posición jurídica es cambiada por un acto. No obstante existe una diferencia fundamental entre, por una parte, actos no permitidos, delitos, cambios de domicilio y el establecimiento de la producción de bienes de consumo, y por la otra, los contratos y la expedición de leyes, como casos paradigmáticos del ejercicio de competencia. La pregunta es, dónde se encuentra esa diferencia. Tres respuestas deben ser examinadas en relación a este punto.

\subsubsection{Declaración (enunciation) e invocación (invocation)}

Esta primera respuesta se encuentra en la tercera diferencia específica, según la cual el cambio de la posición es producido «through and in accordance with enunciations to this effect» $(1968,130)$. Esta formulación expresa el carácter declarativo o de declaratividad de la competencia. Lo que debe ser entendido conforme a esta tercera

\footnotetext{
12 Comp. SpaAK (p. 6), 80 y ss.; Alexy (p. 4), 212 y ss.

13 KeLsEn, Reine Rechtslehre, 2. ${ }^{a}$ ed., 1960, 150 y ss.

14 RaZ, Practical Reason and Norms, 1975, 102.

15 MacCormick, H. L. A. Hart, 1981, 74 y ss.
} 
diferencia específica, se aclara al interpretar la citada formulación rossiana en su contexto. Dicho contexto es definido mediante la primera diferencia específica, la cual dice que las competencias se fundan en normas o reglas jurídicas. Esto significa que la declaración por sí misma no provoca los efectos jurídicos. Ella produce éstos porque existen reglas que producen los efectos jurídicos según la declaración. KELSEN describe esto como la transformación por el ordenamiento jurídico del sentido subjetivo de un acto en un sentido objetivo ${ }^{16}$. Para la definición del concepto de competencia, Ross no usa en On Law and Justice la palabra «enunciation», sino la expresión «dispositive declaration» ${ }^{17}$. En lo que concierne a disposiciones privadas, el concepto de disposición es definido así:

It is pronouncement which as a rule -unless there exist particular grounds for invalidity - produces autonomous legal effects, that is, legal effects which the disponent desires to produce (1958, $222 \mathrm{ff}$.$) .$

El concepto de voluntad (desire) desempeña un papel importante en esta definición. Pero la voluntad es un concepto sicológico. Esto conduce a preguntar si Ross en Directives and Norms ha cambiado su terminología con el objeto de expresar un cambio en el objeto, es decir, un cambio de una comprensión sicológica del ejercicio de la competencia a una comprensión lingüística del mismo. No obstante, éste no es el caso. Tanto en Directives and Norms como en On Law and Justice, Ross describe la competencia como un «special case of power» $(1958,166 ; 1968,130)$, y en ambos casos define lo que es designado con «power» con ayuda del concepto de los efectos jurídicos deseados (desired):

Power exists when a person is able to bring about, through his acts (1958, 166: to cause certain) desired legal effects $(1968,130)$.

Debido a que la competencia debe ser un caso especial de poder jurídico (power), la cláusula de voluntad (desire-Klausel) es, en esta definición, aplicable también a la competencia ${ }^{18}$. No hay evidencia alguna que permita afirmar, que un cambio tuvo lugar en este punto.

Como se ha expuesto hasta ahora, la declaración como tal no basta para generar los efectos jurídicos. El acto de habla debe su fuerza a las reglas, sobre las cuales se basa. Ésta es la dimensión objetiva de la competencia. La cláusula de voluntad añade a ella un plano subjetivo. Las competencias son ejercidas regularmente ${ }^{19}$, porque el efecto

16 KeLSEN (p. 13), 152: «Indem die Rechtsordnung durch generelle Norm Individuen ermächtigt, Verträge zu schließen, erbebt sie den subjektiven Sinn des rechtsgeschäftlichen Aktes zu einem objektiven».

17 Ross, On Law and Justice, 1958, 166. En adelante será citado este libro — como Directives and Normsa través del año de impresión y del número de página.

${ }_{18}$ Quizás Ross sustituyó «dispositive declaration» por «enunciation» para evitar el peligro de una contradicción. El peligro de una contradicción surge, porque por una parte la competencia es definida como «power of disposition [...] exercised by a dispositive declaration» $(1958,166)$, mientras por otra parte el concepto de disposición es especialmente utilizado para referirse a disposiciones privadas $(1958,204,216)$. Si el concepto de disposición hubiese sido reservado únicamente para referirse a disposiciones privadas, hubiese sido descartada la posibilidad de competencias públicas. La existencia de dichas competencias es sin embargo un elemento decisivo de la teoría de la competencia de Ross. Este problema es solucionado a través del uso de «enunciation» $(1968,130)$ como una expresión general para la descripción tanto del «public legal act» como de la «private disposition» $(1958,217)$.

${ }_{19}$ A veces las intenciones no son intenciones actuales sino «justifiably imputed intentions»; comp. MACCORMicK (p. 15), 74. 
jurídico es querido y pretendido. Así, la tercera diferencia específica de Ross está caracterizada por la relación entre regla e intención en el acto de habla. Esto coincide con la definición de invocación (Anrufungs- oder Invokationsdefinition) de la competencia de N. MaCCORMicK. Según esta definición es la competencia -MACCORMicK emplea la expresión «power»-

conferred by a rule when the rule contains a condition which is satisfied only by an act performed with the (actual or imputed) intention of invoking the rule ${ }^{20}$.

La declaración o enunciación de Ross debe ser interpretada como un acto de habla que es efectuado con dos intenciones, las cuales son necesarias para la invocación de una regla, según MACCORMICK. La primera consiste en que los efectos jurídicos (actuales o por ella incorporados) sean queridos. La segunda intención consiste en que los destinatarios del acto de habla reconozcan que el acto es efectuado con la primera intención. El ejercicio de la competencia es, en este sentido, un acto público. El público debe consistir en al menos un destinatario. Ross expresa la necesidad de esta segunda intención no de manera explícita. Ella está de manera implícita en su formulación «though and in accordance with enunciations to this effect» $(1968,130)$. Declarar significa aquí hablar a un público que está compuesto al menos, por una persona distinta del autor de la declaración.

Con estos fundamentos puede ser resuelto el problema de la delimitación. La posición jurídica puede ser cambiada de hecho mediante un acto no permitido o un delito. Pero esto no es producido «though and in accordance with enunciations to this effect» $(1968,130)$. Quien mata a un hombre con ciertas intenciones comete homicidio; pero claro estas intenciones no deben ser declaradas para cometerlo. La responsabilidad penal no se funda ni con base ni según cualquiera sea la declaración pública que apunta a ese efecto. Y las intenciones que son exigidas por la definición jurídica de homicidio «do not include an intention that one's act be recognized as one intended to satisfy the conditions for being "murder" ${ }^{21}$.

El criterio de declaración y el de invocación, es decir la declaratividad, sirve no sólo en casos en los cuales se trata de actos no permitidos o delitos. En el caso del cambio de domicilio, aludido por RAZ, nada depende de alguna declaración sobre los efectos jurídicos en el derecho de impuestos o de seguridad social, y tampoco son definidos los cambios de posición jurídica por las intenciones que relacionan a aquél que cambia de domicilio con su cambio ${ }^{22}$. Lo mismo vale para el caso mencionado por MACCORMICK sobre el establecimiento de la producción de bienes de consumo.

\subsubsection{Constitutividad}

La segunda respuesta a la pregunta sobre cómo pueden ser diferenciados los ejercicios de competencia de otros cambios en la posición jurídica, en especial de aquéllos que son producidos por actos ilegales, se relaciona estrechamente con la primera. Ella es formulada por Ross así:

\footnotetext{
20 MACCORMick (p. 15), 74.

21 Ibid.

$22 \operatorname{SPAAK}($ p. 6), 125.
} 
Those enunciations in which competence is exercised are called actes juridiques, or actsin-the-law, or, in private law, dispositive declarations. Examples are: a promise, a will, a judgment, an administrative licence, a statute. An act-in-the-law is, like a move in chess, a buman act which nobody can perform as an exercise of his natural faculties. Norms of competence are, like the rules of games, constitutive (1968, $130 \mathrm{ff}$.).

Actos que sólo son concebibles como actos constituidos por reglas, pueden ser llamados «actos institucionales» («institutionelle Handlungen»). El concepto de actos institucionales ofrece un criterio útil para distinguir los ejercicios de competencia de otros cambios en la posición jurídica; de ser posible, con la suficiente claridad con la cual este concepto permite distinguir entre reglas constitutivas y regulativas. La distinción propuesta por Ross es cercana a aquélla presentada por J. L. SEARLE ${ }^{23}$, quien ha contribuido de manera fundamental a este debate en la filosofía del lenguaje. De acuerdo con ambas dicotomías, la diferencia se encuentra en la relación lógica entre reglas y actos. Ross emplea como ejemplos, el aparcamiento de un vehículo y el juego de ajedrez. El aparcamiento de un vehículo es «an activity whose performance is logically independent of any rules governing it» $(1968,53)^{24}$. El aparcamiento de un vehículo es, en este sentido, una actividad natural (“natural” activity», 1968, 53). Por el contrario, el juego de ajedrez no es una actividad natural. Sin las reglas de dicho juego, no se pueden entender como movimientos de ajedrez, aquellos movimientos de las figuras de madera en un plano cuadrado. E. BULYGIN ha expresado esto de la siguiente manera: «They are constitutive because they define patterns of behaviour, which do not exist outside these rules» ${ }^{25}$.

J. RAZ ha argumentado contra la distinción entre reglas constitutivas y regulativas que «all rules are both constitutive and regulative» ${ }^{26}$. Si esto fuera cierto, colapsaría tal distinción. Ella no podría ser aplicada para diferenciar los ejercicios de competencia de otros actos, en razón a su carácter institucional.

La crítica de RAZ a la dicotomía entre reglas constitutivas y regulativas se basa en la tesis según la cual, se pueden formular dos distintas descripciones de actos con respecto a cada regla; una que no se refiere a la regla y otra que sí se refiere a ésta. RAZ ofrece los siguientes ejemplos:

1(a) «Giving $£ 50$ to Mr Jones»

2(a) «Saying "I promise"» (b) «Paying income tax»

(b) «Promising» 27 .

Quiero añadir un tercer par de descripciones de actos:

3 (a) «Killing people with a bomb»

Las reglas de la promesa son el paradigma de reglas constitutivas. La pregunta debe ser por esto, si 2(a) realmente describe un acto que corresponde a las reglas de

${ }^{23}$ SeArLe, Speech Acts, 1969, 33 ss.

24 SEARLE (p. 23), 34, usa casi las mismas palabras: «Regulative rules regulate a pre-existing activity, an activity whose existence is logically independent of the rules».

${ }_{25}$ Bulygin (p. 2), 211. Comp. HART, The Concept of Law, 2. ${ }^{a}$ ed., 1994, 32: «If such rules of this distinctive kind did not exist we should lack some of the most familiar concepts of social life, since these logically presuppose the existence of such rules». RAZ.

${ }_{26}$ RAZ (p. 14), 109. Comp. tambien SPAAK (p. 6), 122, 178-179, el cual concuerda en este punto con 
manera tal que «could be given regardless of whether or not there is such a rule» ${ }^{28}$. Contra esto puede ser argumentado que la descripción del acto «Saying "I promise"» sería la descripción de un acto incomprensible si no existieran las reglas de la promesa. Si las reglas de la promesa no existieran o fuesen completamente desconocidas por todos los participantes de la comunicación, entonces podría tener tan poco sentido para ellos la descripción del acto «Saying "I promise"», como la descripción del acto «Saying "I Tromise"». Sin presuponer las reglas de la promesa 2(a) no sería un equivalente serio a la proposición 2(b), la cual describe un acto con referencia a las reglas que lo hacen posible. La «pareja» consistiría entonces en la descripción de un acto institucional comprensible y en la descripción de una expresión incomprensible. Algo totalmente diverso ocurre en relación con el caso del asesinato. Aún si fuesen abolidas aquellas reglas que prohíben el homicidio o éstas fuesen olvidadas por todos, todos podrían entender en cualquier caso en qué consiste el acto descrito por $3(\mathrm{a})$ «Killing people with a bomb» ${ }^{29}$. Aquí surte efecto el «teorema de la pareja» de RAZ (Raz' «Paartheorem»), lo que muestra que la regla, a la que se refiere 3(b), es una regla regulativa.

RAz ha aducido un contraargumento contra una objeción similar en algunos aspectos a la que se acaba de presentar. Según ésta, la expresión «I promise» no es ninguna promesa si ella no es hecha con la intención de asumir una obligación. Por ello debe ser sustituida la descripción del acto 2(a) por la descripción «saying "I promise", with the intention of creating a voluntary obligation ${ }^{30}$. Esto presupone la existencia de las reglas de la promesa. La respuesta de RAZ a esta hipotética objeción es:

the revised act description does not presuppose the existence of the rule about promising, it merely presupposes the agent's belief that there is such a rule ${ }^{31}$.

No está completamente claro lo que significa la condición en virtud de la cual el agente debe creer en la existencia de las reglas de la promesa. Si aquél que describe el acto debe conocer las reglas que el agente cree que existen; o por el contrario, si es suficiente saber que el agente se refiere a cualesquiera reglas sin saber cuáles son. La segunda alternativa conduce al campo de la objeción presentada más arriba, según la cual 2(a) describe algo incomprensible y, en este sentido, absurdo si las reglas de la promesa no son presupuestas. La cláusula «with the intention of creating a voluntary obligation» en la descripción revisada, muestra que la persona, cuyo acto es descrito, conoce las reglas de la promesa. Bajo esta condición dicha nueva descripción expresa un acto completamente comprensible y como tal, en este sentido, claramente inteligible; sin embargo, la comprensibilidad cobra un precio demasiado alto al argumento de RAz: la descripción presupone una regla. Que la regla presupuesta no deba de hecho existir, es decir —en la terminología utilizada por RAZ - que la regla presupuesta no deba ser justificada, practicada o promulgada ${ }^{32}$, no tiene ninguna importancia decisiva. Si las reglas de la promesa no valen, entonces aquél que promete, con base en la creencia de que dichas reglas si valen, en realidad intenta prometer. Es imposible describir un intento tal sin que

28 RAZ (p. 14), 109.

293 (a) estaría entonces ligada, mediante el elemento constitutivo del supuesto de hecho «medios que constituyen un peligro público» del numeral segundo del $\$ 211$ del Código Penal Alemán (StBG), no sólo al concepto de homicidio sino también al hecho mismo del homicidio.

30 Raz (p. 14), 109.

31 Ibid.

32 RAZ (p. 14), 80. 
se presupongan las reglas — en este caso no válidas - de la promesa. La revisión de la descripción del acto tiene por tanto como consecuencia que tanto 2(a) como 2(b) presupongan reglas. Éste es un criterio claro que muestra la razón por la cual estas reglas son reglas constitutivas. Se encuentra en RAZ una formulación cercana. RAZ dice que a veces una regla «regulates only actions done with an intention of invoking the rule» ${ }^{33}$. Dicha formulación es similar a la tesis de la invocación de MACCORMICK discutida anteriormente. Un acto que invoca una regla, no puede ser descrito sin referencia a la misma.

Es cierta, después de todo, la tesis de Ross según la cual las normas de competencia son reglas constitutivas; lo que implica que los ejercicios de competencia son actos institucionales. Este criterio está íntimamente relacionado con aquel de la declaración y la invocación. Dicho criterio es él mismo una interpretación de lo que se entiende por declaración e invocación.

\subsubsection{Autonomía}

Una tercera respuesta a la pregunta acerca de cómo se pueden diferenciar los ejercicios de competencia de otros cambios en la posición jurídica, fue dada por J. Raz. Según RAZ, la diferencia no se encuentra en las características intrínsecas de los actos de ejercicio de la competencia, ni en las características de la clase de las normas de competencia, sino en los fundamentos para el reconocimiento de competencias. Un acto debe ser considerado un ejercicio de competencia, cuando el fundamento para reconocer la posición cambiada consiste en «that it is desirable to enable people to affect norms and their application in such a way if they desire to do so for this purpose» ${ }^{34}$. Este criterio no compite con los dos criterios anteriormente discutidos. Éste más bien los complementa ${ }^{35}$.

Algo similar a este criterio se encuentra en Ross ${ }^{36}$. Él describe los fundamentos de la competencia privada en estos términos:

The social function of private autonomy is to enable the individual to shape his own legal relationships, in accordance with his own interests, within the framework of the legal order $(1969,132)$.

Esto corresponde al criterio de RAz de la deseabilidad (Erwünschtheit), el cual permite al particular influir en la posición jurídica tanto como quiera. En el derecho público las cosas ocurren de otra forma. La opinión de Ross es contundente en este punto. No es conveniente que los poderes públicos actúen como quieran. Ellos deben actuar en función del interés público:

the competence bestowed upon the competent person for the protection not of his own interests but of the interests of a community $(1958,203)$.

Esto muestra que la autonomía privada no es el fundamento único y fundamental de la competencia. La autonomía pública es el segundo ${ }^{37}$.

$33 \operatorname{RaZ}$ (p. 14), 110.

34 RAZ (p. 14), 102.

35 Comp. SPAAK (p. 6), 124

36 Comp. tambien HaRT (p. 25), 34, el cual habla de «desirable social activities».

37 Comp. Habermas, Faktizität und Geltung, 4. . ed., 1994, 161; AlEXY, «Grundrechte und Demokratie en Jürgen Habermas' prozeduralem Rechtsparadigma», en BEHRENDS y DREIER (eds.), Gerechtigkeit und Geschichte, Beiträge eines Symposions zum 65. Geburstag von Malte Dießelhorst, 1996, 78. 
De esta forma han sido presentadas las características principales del concepto de competencia de Ross. Naturalmente permanecen abiertas numerosas preguntas. Éstas se refieren por ejemplo, a la clasificación rossiana de las competencias en personales, procedurales y sustanciales $(1968,130)$; su diferenciación, recientemente nombrada, entre competencias privadas y públicas (1958, $202 \mathrm{ff.,} 217 \mathrm{ff}$.; 1968, $132 \mathrm{ff}$.); la relación entre el concepto de competencia y el de invalidez $(1968,131)$; la construcción rossiana de los derechos fundamentales $(1968,134)$; el concepto de poder o poder jurídico («power»), el cual, como ya se mencionó, Ross considera como más general y contrapuesto a la noción de competencia $(1958,166 ; 1968,41,130)$; y la pregunta acerca de cómo las normas de competencia, que constituyen la más alta autoridad de un sistema jurídico, pueden como tal ser cambiadas ${ }^{38}$. La investigación sobre todos estos temas profundizaría y enriquecería seguramente el modelo del concepto rossiano de competencia. Esta investigación sin embargo no es necesaria para comprender su naturaleza. Su naturaleza puede ser comprendida con ayuda de los conceptos de posibilidad, normatividad, dispositividad y declaratividad. Los conceptos de constitutividad y de autonomía constituyen algo así como el fondo teórico. RAZ describe el análisis del concepto de competencia de Ross como «on the whole unsuccessful» ${ }^{39}$. Nuestra reconstrucción muestra la verdad de lo contrario.

\section{EL PROBLEMA DE LA REDUCCIÓN}

El problema de la reducción tiene dos aspectos. El primero de ellos se refiere a la pregunta acerca de si el concepto de competencia puede ser reducido a otros conceptos. El segundo aspecto se refiere a la pregunta acerca de si las normas de competencia pueden ser reducidas a otras normas, en especial a las obligatorias. Ambas cuestiones están estrechamente relacionadas. Ellas sin embargo deben ser tratadas de manera separada, porque no se puede descartar que a ellas correspondan respuestas distintas entre sí.

\subsection{El teorema de la reducción de Ross}

La parte más interesante de la teoría de la competencia de Ross es su tesis según la cual, el concepto de competencia puede ser reducido al concepto de obligación. Esta tesis se inserta en una más amplia, por la cual se entiende:

that «obligation» is the fundamental directive category in which any norm may be expressed $(1968,117)$.

Esta tesis implica que todo concepto normativo puede ser reducido al concepto de obligación. Esta tesis debe ser denominada como teorema de la reducción ( «Reduktionstheorem»). La reducción de todo concepto normativo al concepto de obligación se realiza a través de tres operaciones, que pueden denominarse como «negación» (Negierung), «relativización» (Relativisierung) y «potencialización»(Potentialisierung).

\footnotetext{
38 Comp. Ross, «On Self-Referece and a Puzzle in Constitutional Law», Mind, 78 (1969), 1.

39 RAZ (p. 14), 185.
} 
Sólo el primero de estos términos fue usado expresamente por Ross. Los otros dos serán empleados aquí para hacer explícito lo que está de manera implícita contenido en su teoría. Antes de proceder a la descripción de las tres operaciones, es recomendable echar un vistazo al punto arquimédico del sistema: el concepto de obligación.

\subsubsection{Obligación}

Ross es completamente consciente del hecho de que un número considerable de expresiones normativas se emplean en discursos jurídicos, morales y otros discursos de tipo normativo $(1968,116$ ff.). Él caracteriza su enfoque como una «estilización» (1968, 125, comp. también 117) o formalización (1968, 120 ff.) del lenguaje normativo. La formalización se realiza con ayuda del conocido operador deóntico «O»:

$\ll O(p) »$ means then, the directive which directs that the subject is under an obligation (or is commanded, is advised) to behave in such a way that p becomes true $(1968,155)$.

La equiparación de «is under an obligation»e «is commanded» realizada en este enunciado, indica que Ross relaciona el concepto de obligación (obligation) con el mandato de hacer algo, y no con la prohibición, es decir con la obligación o el mandato de abstenerse de hacer algo; lo que sería en todo caso posible de acuerdo con el sentido de la palabra obligación. Esto es perfectamente claro, debido al hecho de que Ross equipara la obligación de realizar el comportamiento C («obligation $C »)$ con el «command» de hacer $\mathrm{C}$, que es entendido aquí como la denominación de una modalidad deóntica: «command $\mathrm{C}=$ def. obligation $\mathrm{C}$ », $\mathrm{y}$ en consecuencia: «probibition $\mathrm{C}=\mathrm{def}$. obligation not-C» $(1968,127)$. En vez de «obligación» (Verpflichtung) debe hablarse por esto en lo sucesivo más bien de «mandato» (Gebot). Ambas expresiones son representadas por el operador deóntico «O».

Sin embargo, no podemos estar completamente seguros acerca del hecho de que la intención de Ross sea la de separar completamente el concepto de obligación, el cual emplea en su teorema de la reducción, de aquello que él denomina como «existential basis of norms», es decir el «feeling of validity and obligation» $(1968,117)$. El argumento de Ross según el cual las expresiones deónticas fundamentales, que la estilización del lenguaje normativo hace posible, reflejan («mirror») el sentimiento de validez y obligación, parece hablar en contra de una separación del concepto de obligación del concepto de validez. Por otra parte sin embargo, Ross subraya que el concepto de obligación estilizado o formalizado debe ser entendido como:

the directive operator of a norm, that is, for the element which indicates that the action-idea, described by the determination of the subject, situation and theme, is presented as a pattern of behaviour («so it ought to be») (1968, 117).

Esto es una prueba acerca de la ubicación de la obligación estilizada o formalizada en el nivel de las directivas, es decir, en el nivel semántico. El hecho de que Ross ponga de relieve que su concepción estilizada de obligación pueda ser interpretada de manera diversa en:

legal, conventional, and autonomous moral contexts [...] according to the different ways in which norms are felt as valid $(1968,117)$, 
confirma esta apreciación. Finalmente, hay también un argumento sustancial a favor de separar el concepto de obligación del concepto de validez. El teorema de la reducción no depende de ninguna forma del concepto de validez. Nuestra reconstrucción trabajará por estas razones con un concepto de obligación o de mandato separado o libre del concepto de validez, el cual es expresado a través del operador deóntico «O».

Ross describe de tres maneras diversas el estatus del concepto de obligación: como un operador $(1968,117)$, como una modalidad $(1968,118,134)$ y como una categoría $(1968,117)$. Todas estas tres caracterizaciones son correctas. El concepto de operador expresa el rol de la obligación como elemento de la norma (1968, 117); el concepto de modalidad hace hincapié en la integración del concepto de obligación en un sistema de modalidades y el concepto de categoría acertadamente indica el carácter fundamental del concepto de obligación.

Según Ross, el concepto de obligación o de mandato es no sólo un concepto fundamental, sino además la única categoría normativa que no es posible reducir («the single and irreducible normative category», 1968, 118). La tesis de la fundamentalidad es verdadera, si es posible la reducción de todos los otros conceptos normativos al concepto de obligación o mandato. La tesis de la singularidad es verdadera, si no existe ningún otro candidato al cual el teorema de la reducción le sea aplicable. A continuación deben ser consideradas sólo alternativas relativamente triviales, como son el concepto de prohibición y el de permiso. Una alternativa que está lejos de ser trivial, es el concepto de competencia propiamente dicho. S. L. PAULSON ${ }^{40}$ ha resaltado que elementos de esta alternativa se encuentran en KELSEN ${ }^{41}$. Si tal teorema, opuesto al teorema de la reducción, fuese verdadero, la suposición de singularidad e irreductibilidad de Ross debería ser abandonada. No obstante, su tesis de la fundamentalidad sobreviviría y no perdería su importancia. Lo contrario ocurriría si existiese más de un único concepto fundamental al cual el teorema de la reducción le fuera aplicable de una manera no trivial. En este caso serían posibles descripciones de sistemas jurídicos, las cuales describirían a la vez lo mismo y no lo mismo. Debido a que la posibilidad de un teorema opuesto al de reducción no es el punto central que trata A. Ross, tal posibilidad no puede ser discutida aquí.

Si el teorema opuesto al de la reducción fuese correcto, la suposición de la irreductibilidad no sería sin embargo falsa. En este caso, la modalidad de la obligación o del mandato sería reducible a otras modalidades normativas. Esto puede ser denominado como el problema interno de la irreductibilidad. El problema de la irreductibilidad externa se refiere a la pregunta acerca de si la modalidad de la obligación puede ser reducida a otros conceptos que no tienen sin embargo el carácter de conceptos normativos, como por ejemplo los conceptos de sentimiento, poder y regularidad. Existen buenos argumentos que permiten sostener la imposibilidad de una tal reducción externa, pero tales argumentos no pueden ser tampoco investigados aquí. Nos limitaremos a la reductibilidad dentro de la familia de los conceptos normativos.

\footnotetext{
40 Paulson, «An Empowerment Theory of Legal Norms», Ratio Juris, 1 (1988), 58, 67 y ss.

41 Kelsen (p. 13), 80 y ss.
} 


\subsubsection{Negación}

El primer paso de la reducción es realizado con ayuda de la negación (Negierung oder Negation). Esta operación es muy simple. Consiste en la definición de la prohibición de $p(F p)$ a través de la obligación de no hacer $p$, es decir, mediante el mandato de no- $p(O \neg p)(1968,116,127)$. Además hace parte de esta operación la definición del permiso de $p(P p)$ mediante la negación del mandato de no- $p(\neg O \neg p)$. Por último, dicha operación incluye también la definición del permiso de no- $p(P \neg p)$ a través de la negación del mandato de $p(\neg O p)(1968,128)$. Nada de esto es nuevo y todo ha sido descrito con ayuda del cuadrado de oposiciones deónticas (deontisches Quadrat) ${ }^{42}$.

La reducción de la prohibición y del permiso al mandato, mediante la negación interna y externa es una reducción en sentido estricto. Es una «logical transformation(s)... without change of meaning» $(1968,120)$. La mutua definibilidad conduce sin embargo a un problema. Si el mandato, la prohibición y el permiso son modalidades sin excepción mutuamente definibles, surge la pregunta acerca de si una de éstas puede ser la única modalidad fundamental e irreducible; y si esta pregunta puede ser respondida de manera afirmativa, es necesario preguntar entonces, cuál de estas modalidades sería dicha modalidad. La respuesta de Ross se encuentra en el siguiente enunciado:

If there were no negative norms of permission, norms, that is, which state what is not permitted or what the agent is under an obligation not to do, then there would be no normative meaning whatsoever $(1968,120)$.

Este enunciado aborda tres puntos importantes. El primero de ellos se refiere al hecho de que para Ross es de poca importancia, en relación al problema de la reducción, el carácter fundamental de la modalidad del mandato o de la prohibición. Parece ser que todo lo que cuenta para él, es que no se encuentre negación alguna que sea anterior al operador deóntico que expresa la obligación; es decir que, según la terminología utilizada por él, lo que cuenta es que no se presente negación externa alguna al operador deóntico (1968, 150 ff.). El segundo punto hace referencia al hecho de que para Ross, desde un punto de vista técnico e incluso frente a la alternativa, es indiferente definir las normas de prohibición como «normas negativas de permiso» («negative norms of permission», 1968, 120); en otras palabras, para Ross es indiferente definir prohibición como permiso negativo externo. Esto conduce al tercer punto. Ross dice que la modalidad de la obligación o del mandato no es fundamental en un sentido lógico sino en un sentido sistemático. La fundamentalidad sistemática se basa en el hecho de que un sistema que esté compuesto sólo por permisos no es ningún sistema jurídico, salvo que se use la expresión en un sentido abiertamente extraño. El contenido normativo de un tal estado de naturaleza hobbesiano perfecto, sería aquel en el cual ningún contenido normativo existiese. Desde un punto de vista lógico, las tres modalidades normativas, es decir, aquéllas del mandato, de la prohibición y del permiso, son igualmente fundamentales, en la medida en que son recíprocamente reducibles. Desde el punto de vista sistemático son fundamentales por el contrario tan sólo el mandato y la prohibición. De estas dos modalidades, el mandato tiene un estatus especial que no se basa en la

42 Alexy (p. 4), 184. 
lógica, sino en el hecho de que no existe otra alternativa más que el operador deóntico $(O)$ junto con la expresión «mandado no» ( «gesollt nicht» $O \neg$ ), para expresar fácil y naturalmente tanto el mandato como la prohibición. Esto es una especie de fundamentalidad estética. Por esto, no son sólo los fundamentos sistemáticos y los lógicos los que hacen que el mandato sea la verdadera modalidad fundamental deóntica; son los fundamentos sistemáticos junto con los estéticos los que hacen que esta modalidad sea la modalidad por medio de la cual a través de la negación interna y externa, las demás modalidades deónticas pueden ser creadas. La tesis de Ross acerca de la fundamentalidad de la obligación en la forma del mandato es por esto verdadera, aún cuando requiera ser todavía más refinada.

\subsubsection{Relativización}

El siguiente paso consiste en la reducción del concepto de derecho subjetivo ${ }^{43}$ al concepto de obligación. Ross apoya su reducción sobre la idea de HoHFELD en virtud de la cual los derechos o pretensiones son sinónimos correlativos de las obligaciones o deberes $^{44}$. $a$ tiene un derecho en relación con $b$ a $G$ si y solo si $b$ tiene un deber respecto a $a$ en relación a $G$. Lo anterior puede ser expresado a través de la equivalencia

$$
R a b G \leftrightarrow O b a G
$$

«R» significa una relación de derecho triádica (dreistellige Rechte-Relation) ${ }^{45}$. La equivalencia de «RabG» y «ObaG» resulta del hecho de que ambas relaciones, con respecto a $a$ y $b$, son relaciones conversas ${ }^{46}$. Esto significa que de la misma forma, «O» en «ObaG» debe entenderse como una relación. Ross subraya con razón, que para su teorema de la reducción es necesaria la correlatividad en forma de sinonimia, sobre la cual se basa el carácter converso de ambas relaciones $(1968,119)$. Sin embargo, él se preocupa poco del paso del concepto de obligación (Begriff der Verpflichtung) a la relación de obligación (Relation der Verpflichtung), el cual es necesario para la correlatividad. Él dice sencillamente:

an obligation is normally a relation between two persons, that is, A's obligation is normally an obligation to another person B $(1968,118)$.

Esta observación acerca de lo que «normalmente» es el caso, es correcta en relación con el derecho privado, pero no en relación con el derecho público. Ross mismo sostiene:

${ }^{43} \mathrm{La}$ expresión «derecho subjetivo» («subjektives Recht») utilizada en estricto sentido es equivalente a la expresión «derecho a algo» («Recht auf etwas») o «pretensión» («Anspruch») (comp. BENTHAM, Of laws in General, Aut. HART (ed.), 1970, 57 y ss., el cual habla de un «right to services»), usada en un sentido amplio, esta expresión incluye tanto derechos a algo (Rechte auf etwas), como libertades (Freibeiten) y competencias (Kompetenzen) [comp. Alexy (pp. 4), 171 y ss.], y empleada en un sentido específico se refiere a posiciones jurídicas complejas (komplexe Rechtspositionen) como aquella del propietario (Eigentümer), las cuales están compuestas por un haz de derechos a algo, libertades y competencias. En este punto, esta expresión es utilizada en estricto sentido.

${ }^{44}$ HoHFeld, «Some Fundamental Legal Conceptions as Applied», en Judicial Reasoning, en: ders., Fundamental Legal Conceptions as Applied in Judicial Reasoning and Other Legal Essays, 1923, 36 y ss.

${ }^{45}$ AleXY (p. 4), 186

${ }^{46} \operatorname{Alexy}($ p. 4), 190. 
It follows that those obligations which are upheld solely by public prosecution under the threat of penalty are absolute, that is, no claim corresponds to them with regard either to the party whose interests have been injured, or to the state $(1968,128)$.

Tales obligaciones objetivas o como Ross mismo las llama «absolutas» pueden ser formalizadas mediante

\section{Op}

Para pasar de «Op» a «OabG» debe ser relativizado el operador deóntico y con ello también la modalidad del mandato o de la obligación. La relativización del deber ser (Sollen) hace que se pase del sistema de obligaciones objetivas a aquél de las subjetivas. Tan pronto como sea realizada esta operación, salvo aquellas relaciones que conciernen a la competencia, toda relación descrita por HOHFELD puede ser fácilmente creada mediante la introducción de relaciones conversas y sus negaciones ${ }^{47}$. Ross no hace explícita la necesidad de la operación de la relativización, pero ella está implícitamente contenida en lo que él dice.

De manera diversa a la negación, la operación de la relativización no es simétrica. «OabG» implica «OG», pero «OG» no implica «OabG» ${ }^{48}$. Esto deja claro que el concepto de reducción no es idéntico al concepto de equivalencia lógica. «OabG» tiene el mismo contenido normativo que «OG», pero algo ha sido añadido a esta primera expresión: la relativización del contenido.

\subsubsection{Potencialización}

El tercer paso consiste en la reducción de la modalidad de la competencia a la modalidad de la obligación en la forma del mandato. Según Ross, las competencias son reducibles a obligaciones en razón a que:

Norms of competence are logically reducible to norms of conduct in this way: norms of competence make it obligatory to act according to the norms of conduct which have been created according to the procedure laid down in them $(1968,118)$.

A primera vista este argumento no permite sostener la reductibilidad de la modalidad de la competencia, sino afirmar la reductibilidad de las normas de competencia. Sin embargo, dicho argumento se refiere de manera indirecta a la reductibilidad de dicha modalidad.

Las normas de conducta («norms of conduct») se caracterizan por expresar directamente obligaciones. Ross diferencia entre dos formas de normas de conducta: primarias y secundarias. Las normas primarias son «addressed to the citizens stating their obligations»; las normas secundarias son «addressed to judges, directing them to decide certain cases in certain ways» $(1968,92)$. Ross resalta sin duda al mismo tiempo que «(f)rom a logical point of view... there exists only one set of rules, namely, the so called "secondary" rules». El fundamento de ello radica en que las normas primarias se encuentran de manera implícita en las normas secundarias, «whereas the converse

\footnotetext{
47 Alexy (p. 4), 192.

48 Comp. Alexy (p. 4), 186 y ss.
} 
does not hold» $(1968,92 ;$ comp. 1958, 33). Esta reducción dentro de la categoría de la obligación no es de interés aquí. En este punto nos debemos preguntar más bien, si la competencia puede ser reducida a la obligación, bien en normas primarias o bien en normas secundarias, tal como Ross las define.

Las normas de comportamiento contienen obligaciones; si no las contuviesen, no serían normas de comportamiento. Ross dice que «norms of competence make it obligatory to act according to the norms of conduct which have been created according to the procedure laid down in them» $(1968,118)$. ¿Esto significa que dos obligaciones son combinadas, si el procedimiento establecido en las normas de competencia ha sido llevado a cabo? «A» vale por la ejecución del procedimiento de la forma ordenada por el ordenamiento. La estructura fundamental de una norma de competencia reducida puede ser expresada de la siguiente forma, a través de una norma con una doble obligación:

$$
\text { (1) } A \rightarrow O O p
$$

Una alternativa a esta forma es aquella que expresa que la ejecución, de acuerdo con el ordenamiento, del procedimiento fijado en la norma de competencia hace que entre en vigor la norma primaria de conducta. «G» significa validez. La norma de competencia reducida puede ser expresada entonces como se muestra a continuación:

$$
\text { (2) } A \rightarrow G O p
$$

Una pregunta difícil de responder es cómo se comportan (1) y (2) entre sí y cuál de estas formas es la más adecuada. Por fortuna no debe ser respondida esta pregunta para resolver nuestro problema de la reducción. Lo único que importa aquí es que del ejercicio de la competencia $(A)$, de acuerdo con el ordenamiento, resulta una obligación. Dicho ejercicio de la competencia puede ser pensado ya como una obligación, como la satisfacción de una obligación o como la validez de una obligación.

En este contexto puede ser llevado a cabo el paso de la norma de competencia a la modalidad de competencia. Ni $A \rightarrow O O p$, ni $A \rightarrow G O p$ expresan que el concepto de posibilidad es la base del concepto de competencia. Sólo si el titular de una competencia hace uso de ella, las obligaciones son creadas ${ }^{49}$. Por esto, la relación entre el concepto de competencia y el de obligación no se agota a través de las obligaciones que son creadas de hecho con cada ejercicio de competencia. El contenido normativo de una competencia consiste en la clase de las obligaciones que son posibles con base en ella $^{50}$. Por ello, las competencias pueden ser descritas como obligaciones potenciales ${ }^{51}$. Ésta es la razón por la cual, la tercera operación de reducción se llama «potencialización» («Potentialisierung»).

La relación entre el concepto de competencia y aquel de obligaciones potenciales es tan poco simétrica como aquella que existe entre el mandato relativo y el no relativo (relativisiertes und nichtrelativisiertes Gebot). Las competencias implican obliga-

49 Comp. SPAAK (p. 6), 93 y ss.

$50 \operatorname{Alexy}($ p. 4), 218.

51 Esta idea se refiere no sólo a aquellas obligaciones que pueden ser creadas directamente a través de un ejercicio de competencia, sino también a aquellas obligaciones que pueden ser creadas de manera indirecta con base en una competencia y a través de un ejercicio de competencia. Además esta idea se refiere no sólo a mandatos simples (einfache Gebote), sino a todas las modalidades normativas. 
ciones potenciales, pero las obligaciones potenciales no implican ninguna competencia. La razón de esto es simple. Las obligaciones pueden ser creadas no sólo a partir de ejercicios de competencia, sino a partir de otros actos como los actos dañosos, los delitos o los cambios de domicilio. Esto pone de relieve que a través de la operación de la potencialización no todo el contenido del concepto de competencia puede ser reducido al concepto de obligación. El análisis de la cláusula «tbrough and in accordance with enunciations to this effect» $(1968,130)$ en la definición de Ross mostró que la esencia de la competencia, consiste en proveer un instrumento jurídico para el ejercicio de la autonomía pública y privada. Esto no se encuentra contenido en el concepto de obligación potencial. De otro lado, como en el caso del legislador público, debe existir la posibilidad de la obligación, cuando con base en la titularidad de la competencia, el ciudadano debe ser un legislador privado («private legislator») $)^{52}$, tal como fue descrito por HART ${ }^{53}$. Esto está contenido en la tercera operación de la reducción.

Resumiendo, puede decirse que no sólo se confirma como profunda y fructífera la definición de competencia de Ross, sino que su teorema de la reducción es en esencia verdadero. La definición y el teorema se complementan de manera recíproca. El teorema de la reducción expresa la dimensión normativa de la competencia. La definición permite reconocer que la competencia jurídica, en tanto que basada en la normatividad y la normatividad que le sirve de base, encuentran su verdadera naturaleza en algo que es aún más grande: en la autonomía.

\section{2. ¿Continuidad o discontinuidad?}

Más atrás se dijo que aunque exista relación entre ambas preguntas, la reducción de la modalidad de la competencia a la modalidad de la obligación debe ser diferenciada de la reducción de las normas de competencia a las normas que imponen obligaciones. Algunos aspectos de esta relación han sido ya tratados. Aquí se echará un vistazo final al problema de la reducción desde la perspectiva de las normas, con ocasión de la tesis de la discontinuidad de BulYgin. Según BulYgin, Ross ha cambiado su posición de manera radical entre 1958 y 1968. En On Law and Justice, Ross ha defendido una perspectiva reduccionista, según la cual las normas de competencia son reducibles a normas que imponen obligaciones ${ }^{54}$; mientras que, en Directives and Norms, Ross ha debido supuestamente cambiar a una posición no reduccionista al interpretar las normas de competencia como reglas constitutivas ${ }^{55}$. Mi tesis es que la tesis de BULYGIN plantea un cambio demasiado radical. Hay una mayor continuidad en Ross que aquella que supone la tesis de BuLYGIN. La continuidad se hace evidente cuando se comparan los enunciados a través de los cuales Ross expresa su tesis acerca de la reductibilidad de las normas de competencia a normas de comportamiento en 1958 y 1968. La formulación de Directives and Norms ha sido ya citada. Ross dice que

52 HART (p. 25), 41.

53 HART (p. 25), 33, lo expresa también al describir las normas de competencia como «recipes for creating duties».

${ }_{54}$ Bulygin (p. 2), 201, 208.

55 Ibid. 
las normas de competencia son lógicamente reducibles («logically reducible») a normas de comportamiento, porque las normas de competencia hacen obligatorio («make it obligatory», 1968, 118) comportarse según dichas normas, las cuales han surgido conforme al ordenamiento según las normas de competencia. En On Law and Justice fue planteada la tesis de la reducción de esta forma:

The second group contains those which create a competence (power, authority) - they are directives to the effect that norms which come into existence in conformity with a declared mode of procedure shall be regarded as norms of conduct $(1958,32)$.

La diferencia concierne tan sólo a la formulación. En 1968 las normas de competencia son caracterizadas como normas que hacen obligatorio actuar de acuerdo con las normas de comportamiento ( «make it obligatory to act according to [...] norms of conduct», 1968, 118); mientras que en 1958, las normas de competencia son denominadas directivas, que a su vez provocan que las normas deban ser consideradas como normas de comportamiento ( directives to the effect that norms [...] shall be regarded as norms of conduct», 1958, 32). Una directiva que cause que determinadas normas deban ser consideradas como normas de comportamiento, es lo mismo que una norma que imponga la obligación de comportarse según esas normas. Lo anterior significa que no existe cambio alguno en la tesis de Ross, es decir, que el contenido normativo de las normas de competencia puede ser reducido a las normas de comportamiento.

Existen dos formas de reducir las normas de competencia a normas de comportamiento, una primitiva y otra sutil. La versión primitiva clasifica las condiciones del ejercicio de competencia de acuerdo al ordenamiento, como elementos constitutivos complementarios del supuesto de hecho de las normas de conducta. De esta forma, las normas de competencia son reducidas a fragmentos de las normas de comportamiento ${ }^{56}$. Esto se puede denominar como la «teoría de la fragmentación» («Fragmenttheorie»). Ross defiende en los enunciados citados arriba una perspectiva mucho más sutil. Según ésta, el modo correcto de reducción de las normas de competencia a normas de comportamiento se basa en el hecho de que las normas de comportamiento tienen el estatus de metarreglas (Metaregeln). Las normas de competencia son normas de comportamiento sobre la formación, de acuerdo al ordenamiento, de estas últimas. Esto se puede denominar como la «teoría de las metareglas» («Metaregeltheorie»).

La teoría de las metarreglas coincide con aquello que no es reducible, lo cual está incluido en el concepto de expedición o creación de normas de acuerdo al ordenamiento. La expedición de normas es un acto institucional. Como se indicó anteriormente, los actos institucionales presuponen reglas constitutivas. Las reglas constitutivas no son reducibles al mismo nivel que las reglas regulativas. En este sentido tiene razón BuLYGIN. Ross ha introducido un elemento importante de irreductibilidad al diferenciar entre reglas regulativas y reglas constitutivas. Esto comprende tanto la autonomía privada como la autonomía pública, las cuales están ligadas a los actos institucionales del contrato y de la legislación. El contenido normativo de la totalidad puede ser descrito a través de una combinación de un objeto y una metanorma de la obligación. Esta

56 Comp. al respecto, HART (p. 25), 35 y ss. 
combinación está presente tanto en la definición de competencia de Ross del año 1958 como en la del año 1968. Se puede decir por ello que existe en Ross continuidad no sólo en relación a la reductibilidad, sino también al carácter específico de la competencia. Esto fue completamente explicado en 1968 y está presente también en la esencia de la posición de Ross de 1958.

(Traducción de Gonzalo Villa Rosas) 